\title{
Synthesis, Characterization and Swelling Characteristics of Graft Copolymerized Isotactic Polypropylene Film
}

\author{
Teena Sehgal $^{1}$ and Sunita Rattan ${ }^{1,2}$ \\ ${ }^{1}$ Department of Chemistry, Amity School of Engineering and Technology, 580, Palam Vihar Road, Bijwasan, New Delhi 110061, India \\ ${ }^{2}$ Department of Chemistry, Amity School of Engineering and Technology, Amity University Uttar Pradesh, Noida 201303 , India
}

Correspondence should be addressed to Sunita Rattan, srattan@amity.edu

Received 5 January 2010; Accepted 5 April 2010

Academic Editor: Yulin Deng

Copyright ( 12010 T. Sehgal and S. Rattan. This is an open access article distributed under the Creative Commons Attribution License, which permits unrestricted use, distribution, and reproduction in any medium, provided the original work is properly cited.

\begin{abstract}
Grafted membranes were prepared through chemical graft copolymerization of methyl methacrylate (MMA) onto isotactic polypropylene film (IPP). The IPP films were grafted with MMA molecules resulting in IPP-g-MMA grafts using benzoyl peroxide as an initiator in an inert nitrogen atmosphere. Using this method, the degree of grafting and morphology could be controlled through the variation of reaction parameters such as initiator concentration, monomer concentration, reaction time, and the reaction temperature. Optimum conditions pertaining to maximum percentage of grafting $(\% \mathrm{G})$ were evaluated as a function of these parameters. Maximum percentage of grafting (50\%) was obtained at $[\mathrm{BPO}]=0.03 \mathrm{M},[\mathrm{MMA}]=10 \% \mathrm{~V} / \mathrm{V}$, and $[$ Reaction Temperature $]=70^{\circ} \mathrm{C}$ in a [Reaction time] of 120 minutes. IPP-g-MMA films were investigated for their swelling behavior. Water-swelling analysis of IPP-g-MMA was carried out as a function of different percentage of grafting, temperatures, and time. Maximum swelling percentage of IPP-g-MMA (92\%) was observed in 8 hours at $60^{\circ} \mathrm{C}$. The evidence of grafting was carried out by Fourier transform spectroscopy (FTIR), atomic force microscopy (AFM), and scanning electron microscopy (SEM) before and after grafting, respectively. The swelling pattern was characterized by two distinct stages, an initial diffusion-controlled fast swelling, followed by a subsequent slower process controlled by the relaxation of polymer fragments. Swelling chrematistics of IPP-g-MMA make it a potentially useful material.
\end{abstract}

\section{Introduction}

As one of the most commonly used plastics, isotactic polypropylene (IPP) has many valuable qualities, such as low cost and versatility. However, IPP is limited in its applications in some important technological fields because of its lack of chemical functionalities, low surface energy, difficulty to dye, poor hygroscopicity, low impact strength, poor compatibility with other polymers, and sensitivity to photo- or thermal oxidation. In order to overcome these disadvantages, a great deal work has been carried out on the modification of IPP, for example, chlorination, hydroperoxidation, and hydrogen abstraction from tertiary carbons, followed by ozonolysis and graft copolymerization. Among all the methods of modifications, graft copolymerization onto IPP [1] offers an effective approach. In principle, chemical graft copolymerization is an attractive method to impart a variety of functional groups to a polymer backbone. This is a promising method for the modification of the chemical and physical properties of polymer surfaces. The modification of polymers has received much attention recently. This can be applied to modify the membrane surfaces, for example, without affecting their bulk properties. It can provide some desirable properties such as biocompatibility, ion exchange, and thermosensitivity. The importance of membrane surface chemistry in deciding the separation characteristics is well recognized [2-4]. The formation of copolymers of various synthetic and natural polymers via graft copolymerization has been extensively studied [5]. Various monomers such as methyl acrylate (MA) and methyl methacrylate (MMA) have been graft copolymerized with numerous polymeric backbones using various initiating systems. Grafting of IPP with methyl methacrylate monomers through chemical methods using benzoyl peroxide as an initiator leads to 


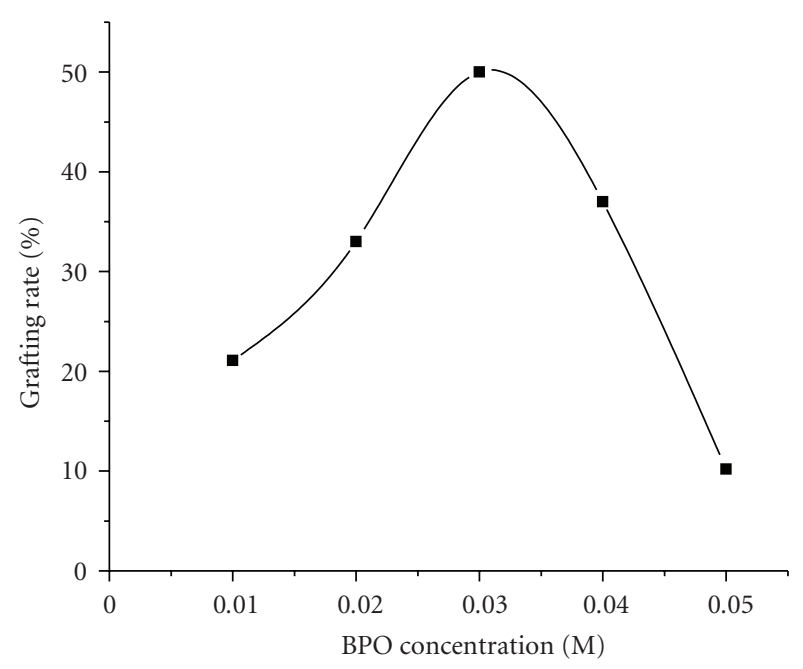

Figure 1: Effect of BPO on Percentage of grafting, [MMA] $=10 \%(\mathrm{~V} / \mathrm{V}) ;[$ Temperature $]=70^{\circ} \mathrm{C} ;[$ Time $]=120$ minutes; [Nitrogen atmosphere] $=20$ minutes; Optimum $[\mathrm{BPO}]=(0.03 \mathrm{M})$.

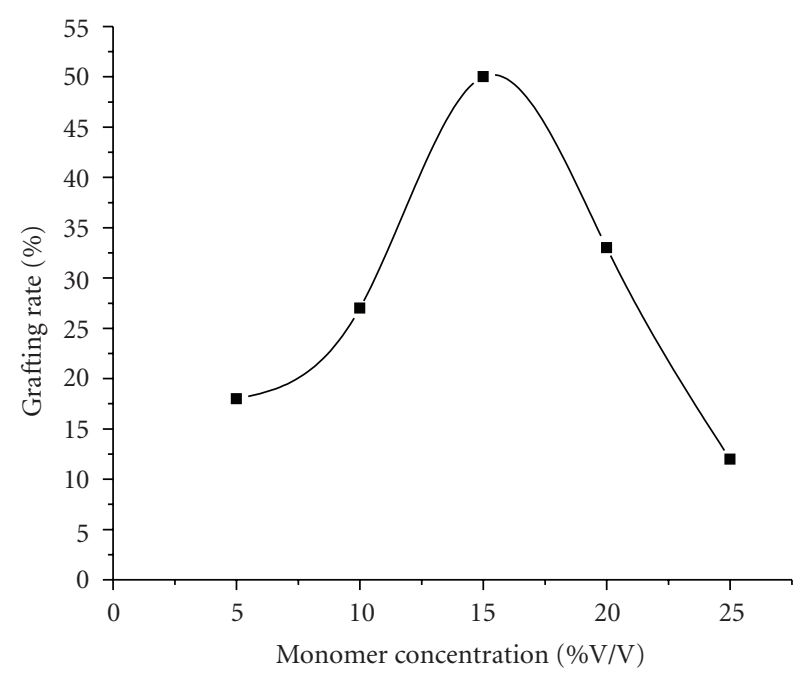

Figure 2: Effect of Monomer Concentration on Percentage of grafting, $[$ Temperature $]=70^{\circ} \mathrm{C}$; $[$ Time $]=120$ minutes; $[\mathrm{BPO}]=$ $0.03 \mathrm{M}$; [Nitrogen atmosphere $]=20$ minutes; Optimum $[\mathrm{MMA}]=$ $10 \%(\mathrm{~V} / \mathrm{V})$.

an appreciable improvement in its properties. Because of its growing commercial applications, IPP film has attracted particular attention. In the present work, an attempt has been made to prepare grafted membrane through grafting of MMA onto IPP through chemical grafting method. We systematically studied the effects of various factors, that is, effect of monomer concentration and initiator concentrations, reaction time and reaction temperature on the grafting percentage. Then, the morphology and structure of the grafted substrates were examined using FTIR, SEM, and AFM. It is investigated that the membranes prepared by grafting of MMA onto IPP films exhibit high water sorption capacity (swelling behavior).

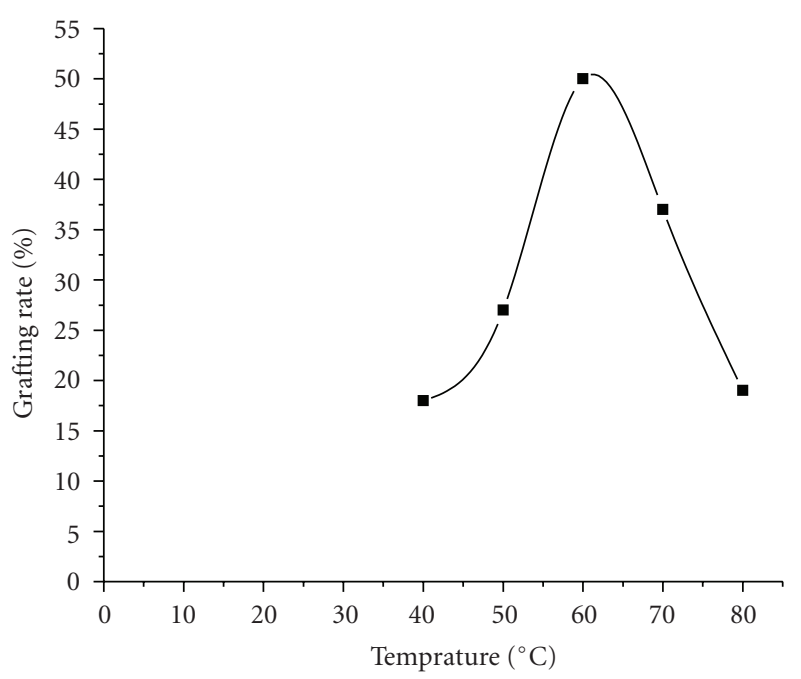

Figure 3: Effect of Temperature on Percentage of grafting, [MMA] $=10 \%(\mathrm{~V} / \mathrm{V}) ;[\mathrm{BPO}]=0.03 \mathrm{M} ;[$ Reaction time $]=120$ minutes; [Nitrogen atmosphere] $=20$ minutes; Optimum [Temperature $]=$ $70^{\circ} \mathrm{C}$.

\section{Experimental}

2.1. Materials. Experiments were performed with commercially available IPP films kindly supplied by Reliance Industries, Ltd. (Noida, India). The IPP films having thickness $(16 \mu \mathrm{m})$ were cut into $2 \times 4 \mathrm{~cm}^{2}$ pieces. IPP films were extensively washed with acetone and dried in a vacuum oven. MMA, BPO, and acetone used in the present work were obtained from Sigma-Aldrich (Stein heim, Germany) and were used without further purification. Nitrogen gas was purified by passing through freshly prepared alkaline pyrogallol solution to remove traces of oxygen.

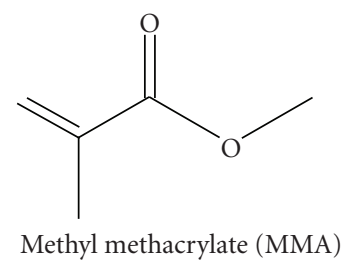

2.2. Grafting Procedure. The grafting reaction was carried out in Pyrex ampoules having a stop cork. Water as a solvent was added first, followed by ferrous sulfate $\left(\mathrm{FeSO}_{4} 1 \times\right.$ $\left.10^{-3} \mathrm{M}\right)$, sulfuric acid $\left(\mathrm{H}_{2} \mathrm{SO}_{4}, 0.1 \mathrm{M}\right)$, initiator $\mathrm{BPO}$ in requisite amount. IPP films were immersed into monomer solution mixture in Pyrex ampoules and purged by bubbling nitrogen for 20 minutes. The grafting reaction was carried out by placing the ampoules in water bath at $60^{\circ} \mathrm{C}$ for 2 hours. After the grafting reaction, the samples were taken out, washed with distilled water at room temperature for 1 hour with stirring and changing of water six times. After the grafting reaction, the samples were taken out from the monomer solution in the ampoules and washed with acetone 


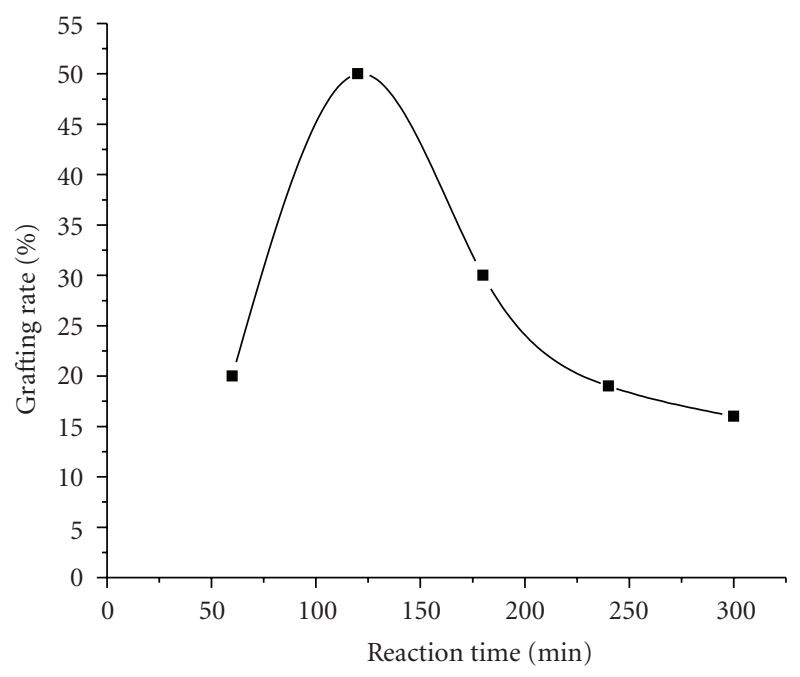

FIGURE 4: Effect of Reaction time on Percentage of grafting, [MMA] $=10 \%(\mathrm{~V} / \mathrm{V}) ;[$ Temperature $]=70^{\circ} \mathrm{C} ;[\mathrm{BPO}]=0.03 \mathrm{M} ;[$ Nitrogen atmosphere $]=20$ minutes; Optimum [Reaction time $]=120$ minutes.

to remove the remaining homopolymer $[6,7]$. The degree of the grafting was determined by the following:

$$
\text { Percentage of grafting }(\% G)=\frac{W_{g}-W_{o}}{W_{o}} \times 100 \text {, }
$$

where $W_{g}$ and $W_{o}$ are the weights of the grafted and ungrafted samples, respectively.

The effects of various reaction parameters such as monomer concentration, initiator concentration, reaction temperature, and reaction time were investigated by this grafting procedure.

\section{Results and Discussion}

The reactionscheme for the graft copolymerization of MMA onto IPP films is shown in Scheme 1. Grafting rate depends upon a large number of reaction parameters [8,9] such as monomer concentration, initiator concentration, reaction temperature, and reaction time were investigated. Therefore, the effects of these reaction parameters were investigated.

3.1. Effect of the Concentration of the Initiator. The effect of the initiator BPO concentration on grafting rate was studied. These results are shown in Figure 1. It was observed that the percentage of grafting increases up to a certain level and reaches a maximum value of $50 \%$ at a concentration of $0.03 \mathrm{M}$ of the initiator, beyond which it decreases slowly. In a grafting point of view, the initial increase is due to the availability of the free radicals generated for grafting of the monomer. When the concentration of the initiator exceeds a certain value, increased free radical concentration results in serious homopolymerization and hence lowers the graft copolymerization. Similar results reported for grafting

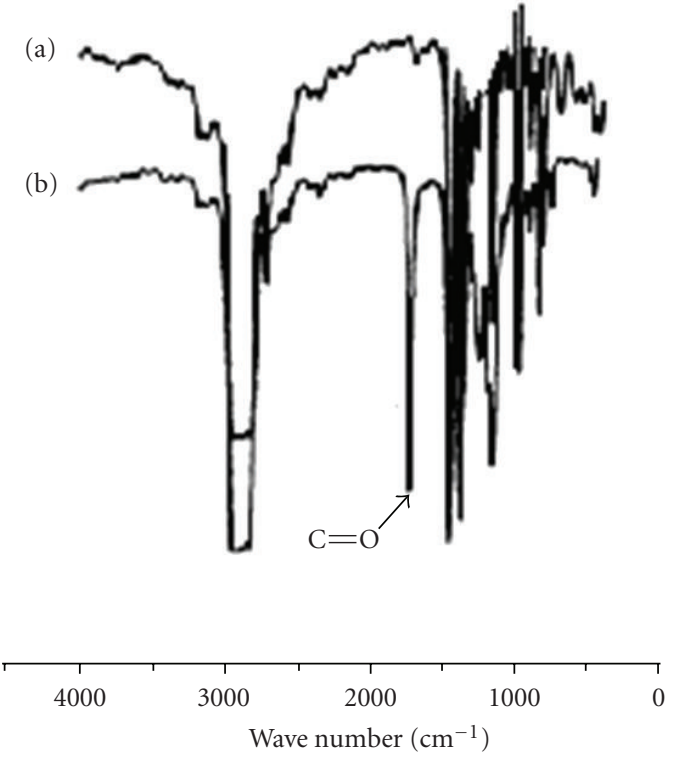

FIGURE 5: FTIR Spectra of (a) Virgin IPP, (b) IPP-g-MMA.

perfluro-2-ethanol acrylic monomer onto poly (ethylene terephthalate) (PET) [10] and for grafting perflouro-2ethanol acrylic monomer/stearyl methacrylate monomer mixture on PET fibers [11].

3.2. Effect of Monomer Concentration. Figure 2 represents the effect of monomer (MMA) concentration on the percentage of grafting ontoIPP film. It can be seen that the grafting percentage increases initially with increasing monomer concentration giving maximum percentage $(50 \%)$ at monomer concentration of $10 \%(\mathrm{~V} / \mathrm{V})$ and then decreases on further increasing the concentration of MMA. The initial increase in grafting may be due to the reason that most of the monomer is utilized by the available free radical sites on the IPP backbone. However, it can be noted that the grafting rate does not exceed beyond the optimum monomer concentration. Also at higher concentrations, the degree of homopolymerization increases, and the grafting percentage decreases. Moreover at the lower concentration, the extent of homopolymerization of the monomer is smaller. The grafting reaches a maximum value and thereafter decreases. This is because the number of free radical sites available on the IPP backbone becomes a limiting factor, and the rate of diffusion is progressively affected by deposition on the polymer backbone [10-12].

3.3. Effect of Temperature. The temperature of grafting medium is one of the most important reaction parameters that have a strong effect on the grafting yield during grafted membrane preparation. The effect of reaction temperature on the graft polymerization was studied at temperature varying from $40^{\circ} \mathrm{C}$ to $80^{\circ} \mathrm{C}$. Generally, the increase in temperature of the grafting mixture up to $70^{\circ} \mathrm{C}$ initially increases the degree of grafting as shown in Figure 3. This may be due to the increase of the initiation and propagation 


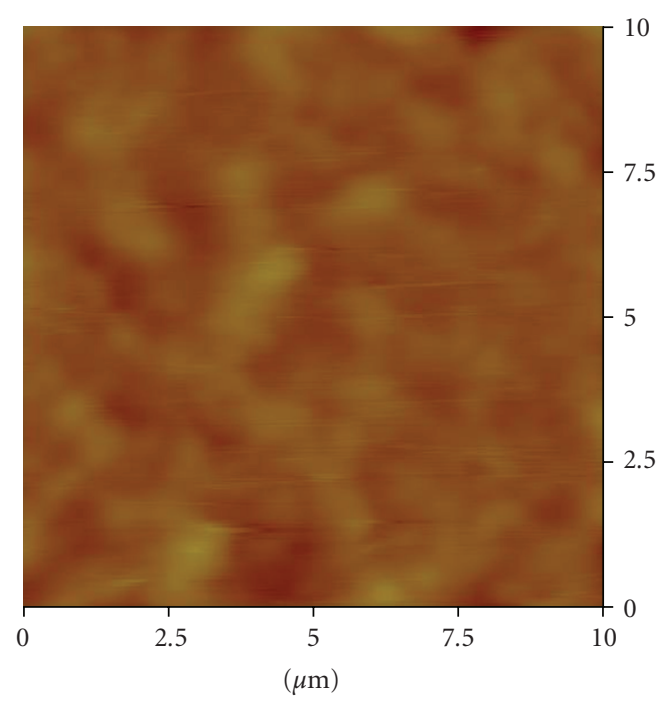

(a)

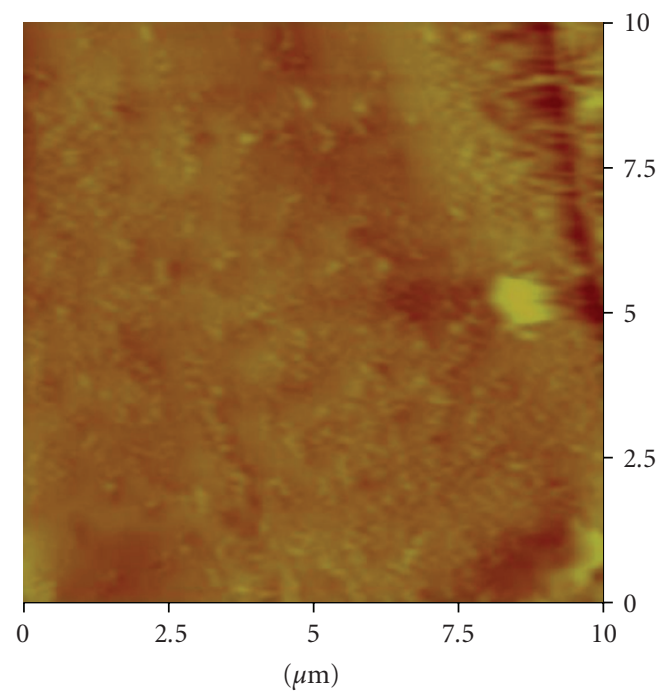

(b)

Figure 6: AFM Images of (a) Virgin PP (b) IPP-g-MMA.

rates of graft copolymerization. Increase in the degree of grafting with increasing temperature is also due to increase in the decomposition of the initiator leading to the formation of more free radicals and the generation of active sites on the polymeric backbone. But beyond $70^{\circ} \mathrm{C}$, the grafting rate decreases, and the film becomes brittle. Due to this, the effect was not studied beyond $70^{\circ} \mathrm{C}$. Probably this is because at higher temperature, higher combination rates of monomer are obtained increasing homopolymerization reactions, which results in a decreased grafting rate $[12$, 13]. Decreased in percentage of grafting beyond optimum temperature is attributed to premature termination of the growing polymeric chains and to the occurrence of chain transfer reactions. Similar observations have been noted by Somanathan et al. with grafting methacrylic acid onto PET using Benzoyl peroxide as initiator [14].

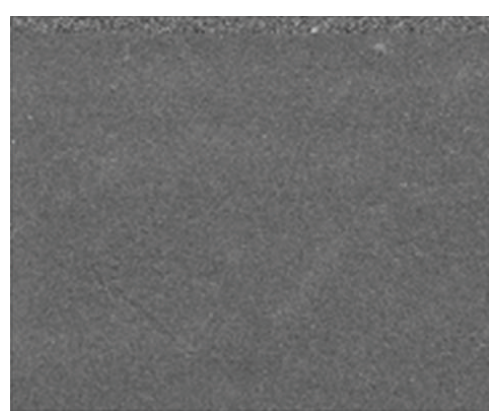

(a)

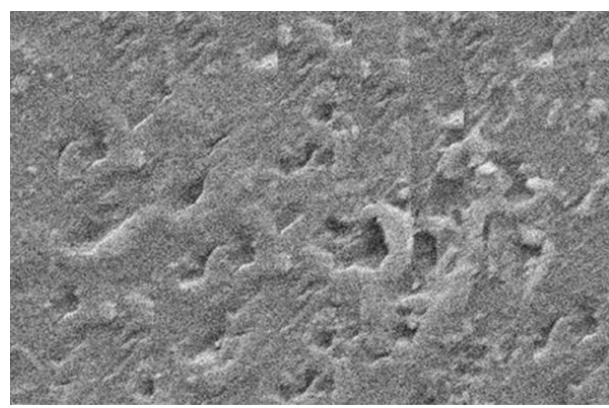

(b)

Figure 7: SEM Images of (a) Virgin PP (b) IPP-g-MMA at X 1000.

3.4. Effect of Reaction Time. It can be seen from Figure 4 that the grafting level increases sharply with increase in reaction time from 60 minutes to 120 minutes. The graft copolymerization rate increases to a maximum value of 120 minutes. With an increase in reaction time, the free radicals have more time for reaction and therefore results in higher level of grafting. After some time, all the initiators and monomers are used up. Thus no further change in grafting level was observed with increasing reaction time. The decrease in grafting may be due to the induced decomposition of the initiator leading to decrease in the concentration of the initiator and hence decrease in active radicals required to generate active sites on polymeric backbone. Similar observations were reported with grafting acrylic monomer such as acrylamide and glycidyl methacrylate on polyamide fibres using other initiators $[15,16]$.

\section{Characterization of Grafted Films: Evidences of Grafting}

FTIR. FTIR spectra of ungrafted and grafted films were recorded on a Perkin Elmer FTIR RX1 $\left(4000-500 \mathrm{~cm}^{-1}\right)$ spectrophotometer (Norwalk, CT) with a $4.0 \mathrm{~cm}^{-1}$ resolution.

\section{Morphological Studies:}

(i) AFM Surface topography of ungrafted and grafted films were performed on a multimode Nanoscope IIIa SPM. Since the polymers are soft materials, tapping mode was used with RTESP tip and a low force constant. 


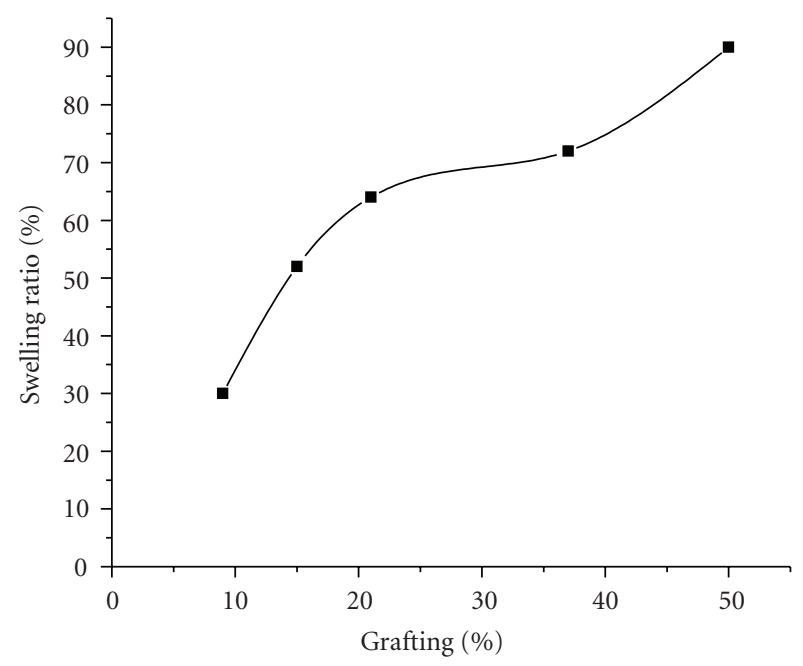

FIGURE 8: Swelling percentage of (IPP-g-MMA) as function of percentage of grafting.

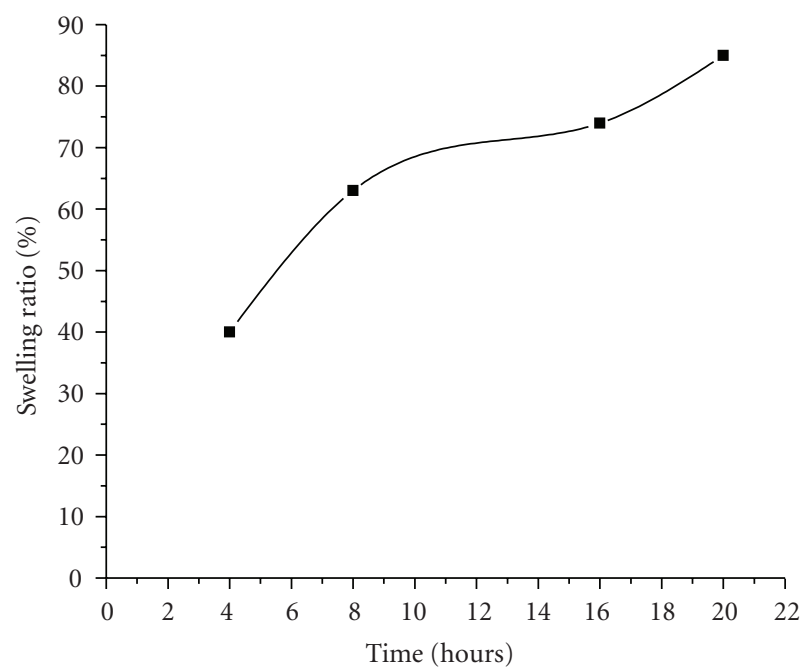

FIGURE 9: Swelling percentage of (IPP-g-MMA) as function of time.

(ii) SEM Surface topology and homogeneity of ungrafted and grafted films were performed on a LEO VP 435 instrument.

4.1. FTIR Analysis. The presence of grafting onto IPP film was confirmed through FTIR analysis. The FTIR spectra of virgin IPP and IPP-g-MMA are shown in Figure 5. The Figure 5(b) shows the stretching bands at $1734 \mathrm{~cm}^{-1}$ for the carbonyl group which is absent in the spectra of virgin IPP 5(a), indicating that MMA has been grafted onto the IPP.

4.2. Morphological Studies. The surfaces of the original IPP and MMA grafted IPP films were examined by AFM and SEM studied, and the results are shown below.

4.2.1. AFM Analysis. In order to elucidate the topological changes under the grafting reactions, the films surfaces were

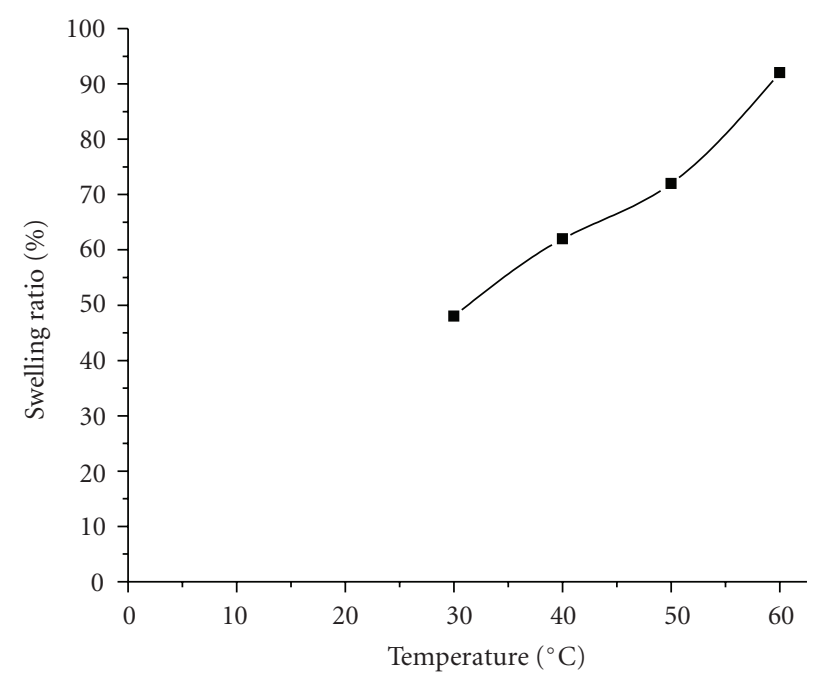

Figure 10: Swelling percentage of (IPP-g-MMA) as function of temperature.

characterized by AFM analysis. Figures 6(a) and 6(b) shows the AFM images of virgin IPP film and the grafted IPP films (IPP-g-MMA). Compared to IPP-g-MMA, virgin IPP presented a smoother surface as shown in Figure 6(a). It is seen that IPP-g-MMA shows continuous roughness as shown in Figure 6(b). The roughness of the grafted film is attributed to the formation of grafted chains on the polymer surface. It can be clearly seen that after surface modification, large quantity of MMA is grafted on the membrane surface.

4.2.2. SEM Analysis. SEM of IPP and IPP-g-MMA at magnification (X 1000) have been taken on LEO VP 435 instrument. The surface was coated with gold to avoid charging under an electron beam. The results are shown in Figure 7. The composition of the grafted IPP with that of pristine IPP shows clearly the change in the topography of the IPP surface. The virgin IPP exhibits a smooth surface pattern whereas the grafted IPP (IPP-g-MMA) exhibits rough surface. The reason for the surface roughness is due to the chemical grafting of MMA onto IPP which opens up its matrix and shows considerable deposition of MMA on the surface of backbone polymer.

\section{Swelling Characteristics}

The swelling ability of a polymer is related to its water permeability and uptake, that is, to its hydrophilicity. The swelling is believed to be a phenomenon similar to osmosis. Swelling is one of the most important parameters affecting on the characteristic properties of the grafted membrane and accordingly determining its applicability for practical use.

The clean, dried grafted films of known weights were immersed in distilled water at $25^{\circ} \mathrm{C}$ until equilibrium was reached (almost 24 hours). The films were removed, 

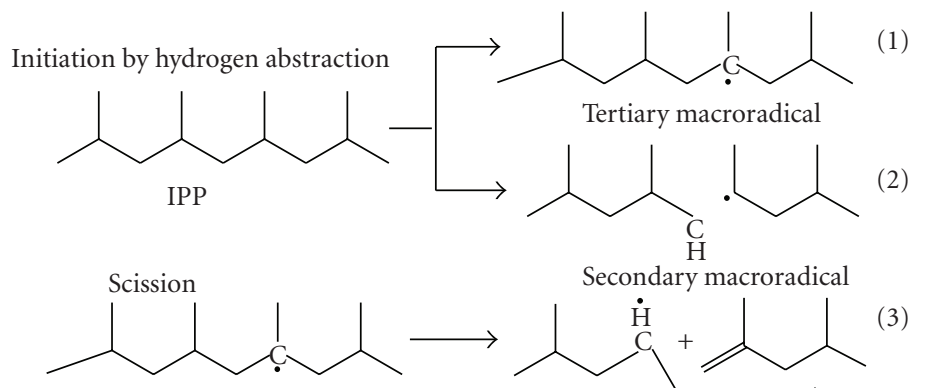

Propagation grafting reactions
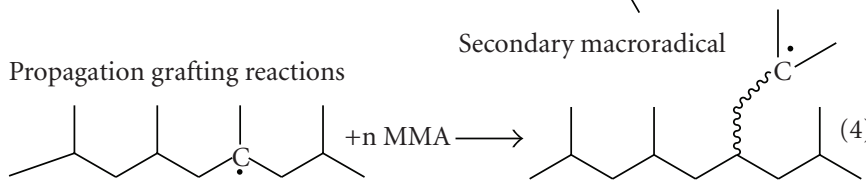

MMA chain growing on IPP<smiles>CCCC(C)[C@@H](C)CC(C)CC(C)CC(C)CC(C)C(C(=C(C)C)C(C)CC(C)C)C(C)CC(C)C</smiles>

MMA chain growing on IPP<smiles>CC#CCC(C)CC(C)C</smiles>

Propagation chain transfer reaction<smiles>CCCC(C)CC(C)CC(C)CC(C)CC(C)CC(C)C</smiles><smiles>CC(C)CC(C)CC(C)(CC(C)C)CC(C)CC(C)CC(C)(C)CC(C)C</smiles><smiles>CC(C)CC(C)CC(C)CC(C)C</smiles><smiles>CC(C)CCC(C(C)CC(C)C)C(C)CC(C)C</smiles><smiles>C=C(C)CC(C)C</smiles>

Scheme 1: Continued. 

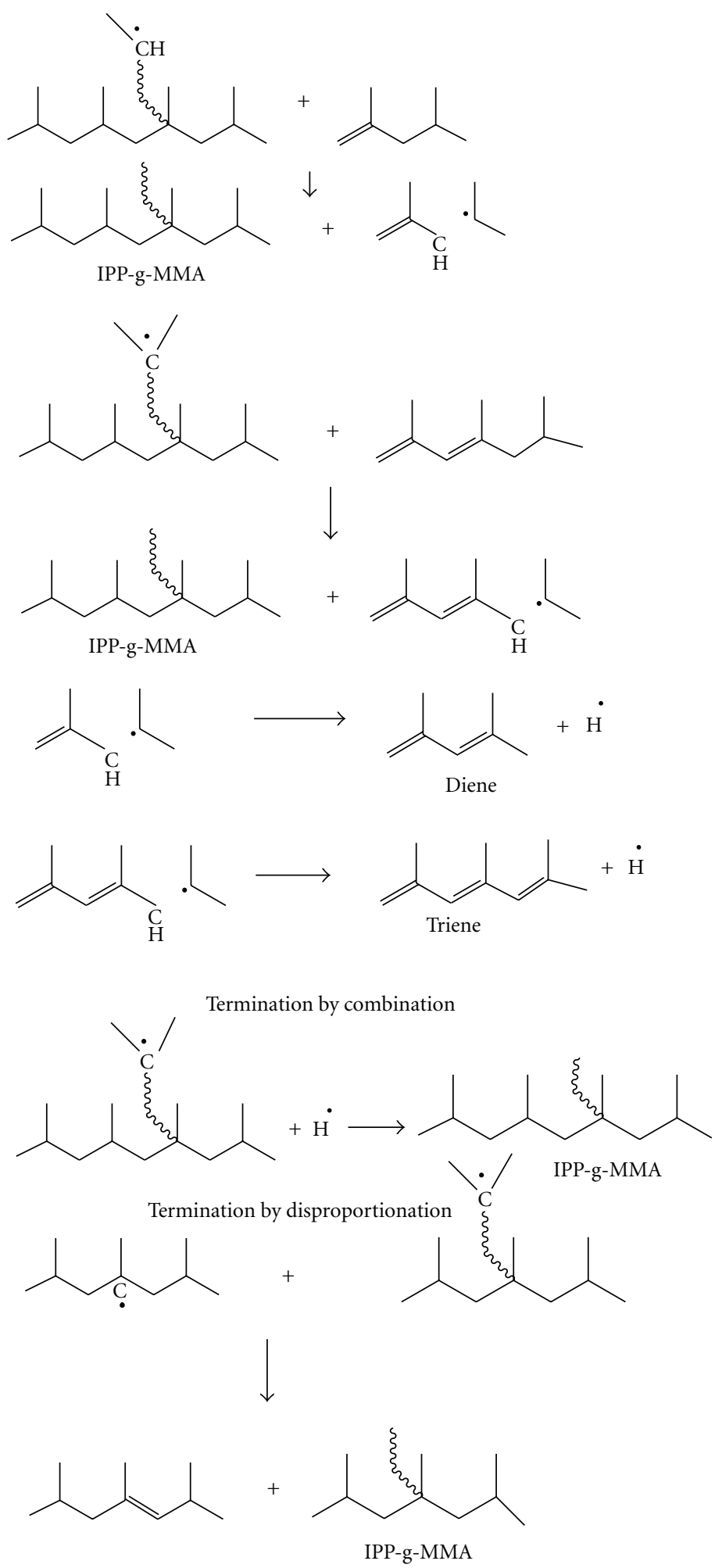

Scheme 1: Mechanism of Grafting copolymerization Process of MMA onto IPP. 
blotted quickly with adsorbent paper, and then weighed. The percentage of amount water uptake was calculated as follows

$$
\text { Swelling Ratio }(\%)=\frac{\left[W_{f}-W_{i}\right]}{W_{i}} \times 100,
$$

where $W_{f}$ is the weight of wet grafted films and $W_{i}$ is the weight of dry grafted films.

\subsection{Swelling as a Function of Percentage of Grafting. Figure 8} shows the swelling of IPP-g-MMA films for different grafting percentage in distilled water. The swelling of the IPP-g-MMA increases with increase in percent grafting. The swelling rate indicates that the initial swelling process is primarily due to the water penetrating into copolymer film through capillary and diffusion. Then the penetrating water is absorbed by functional groups through formation of hydrogen bonds. A higher grafted IPP-g-MMA content results in a greater hydrophilicity which causes a higher swelling ratio.

5.2. Swelling as a Function of Time. Figure 9 shows the swelling behavior of selected samples of high grafting percentage of IPP-g-MMA films as a function of time. It is observed from the table that the grafted samples (IPP-gMMA) show maximum swelling (85\%) in 20 hours in water at temperature of $30^{\circ} \mathrm{C}$. After these conditions the swelling rate dose not increase, indicating that water penetration into copolymer film through capillary and diffusion became less. But further the swelling percentage remains constant on further increasing the temperature.

5.3. Swelling as a Function of Temperature. Figure 10 shows the swelling behavior of selected samples of high grafting percentage of IPP-g-MMA films as a function of temperature. It is observed from the table that the grafted samples (IPP-gMMA) show maximum swelling $(92 \%)$ at $60^{\circ} \mathrm{C}$ in water in time of 8 hours because of the enhancement of diffusion of water into the membrane pores.

\section{Conclusion}

The obtained results show the graft copolymerization of MMA monomer onto IPP film using benzoyl peroxide (BPO) as initiator in a nitrogen atmosphere, this leads to higher grafting yields. Since the efficiency of grafting rate depends on the MMA concentration, BPO concentration, the reaction time, and the temperature of reaction, the grafting yield can be controlled by appropriate selection of grafting conditions. The best conditions for optimum rate of grafting $(50 \%)$ were obtained with a BPO concentration of $0.03 \mathrm{M}$, MMA concentration of $10 \%(\mathrm{~V} / \mathrm{V})$, a reaction temperature of $70^{\circ} \mathrm{C}$, and a reaction time of 120 minutes. The swelling behavior of the grafted films shows a significant increase with increase in percentage grafting. The swelling behavior of the grafted membranes can be regulated by the changing of synthesis conditions. This type of work could encourage the synthesis of new grafted membranes, where some functionality is required, for specific purpose.

\section{References}

[1] H. J. Hayes and T. J. McCarthy, "Maleation of poly(4-methyl1-pentene) using supercritical carbon dioxide," Macromolecules, vol. 31, no. 15, pp. 4813-4819, 1998.

[2] M. Aizawa, "Biofunctional synthetic membranes," in Proceedings of the Materials Science of Synthetic Membranes. Based on a Symposium at the 187th Meeting of the American Chemical Society, D. R. Lloyd, Ed., vol. 269, chapter 1 of ACS Symposium Series, pp. 447-480, American Chemical Society, Washington, DC, USA, December 1985.

[3] D.-M. Wang, T.-T. Wu, F.-C. Lin, J.-Y. Hou, and J.-Y. Lai, "A novel method for controlling the surface morphology of polymeric membranes," Journal of Membrane Science, vol. 169, no. 1, pp. 39-51, 2000.

[4] W. R. Bowen, N. Hilal, R. W. Lovitt, and C. J. Wright, "Characterisation of membrane surfaces: direct measurement of biological adhesion using an atomic force microscope," Journal of Membrane Science, vol. 154, no. 2, pp. 205-212, 1999.

[5] I. Kaur, B. N. Misra, and R. Barsola, "Radiation-induced graft polymerization of vinyl monomers onto polyamide6," Angewandte Makromolekulare Chemie, vol. 234, pp. 1-12, 1996.

[6] Q.-W. Dai, Z.-K. Xu, H.-T. Deng, Z.-M. Liu, J. Wu, and P. Seta, "Surface modification of microporous polypropylene membranes by graft polymerization of N,N-dimethylaminoethyl methacrylate," Chinese Journal of Polymer Science, vol. 22, no. 4, pp. 369-377, 2004.

[7] O. Sainli and E. Pulet, "Solvent-assisted graft copolymerization of acrylamide on poly(ethylene terephthalate) films using benzoyl peroxide initiator," Journal of Applied Polymer Science, vol. 47, no. 1, pp. 1-6, 1993.

[8] C. Makhlouf, S. Marais, and S. Roudesli, "Graft copolymerization of acrylic acid onto polyamide fibers," Applied Surface Science, vol. 253, no. 12, pp. 5521-5528, 2007.

[9] Z. Liu, L. Song, X. Dai, G. Yang, B. Han, and J. Xu, "Grafting of methyl methylacrylate onto isotactic polypropylene film using supercritical $\mathrm{CO}_{2}$ as a swelling agent," Polymer, vol. 43, no. 4, pp. 1183-1188, 2001.

[10] M. Louati, A. El-Achari, A. Ghenaim, and C. Caze, "Graft copolymerization of polyester fibers with a fluorinecontaining monomer," Textile Research Journal, vol. 69, no. 5, pp. 381-387, 1999.

[11] D. Saihi, A. El-Achari, A. Ghenaim, and C. Caze, "Graft copolymerization of a mixture of perfluorooctyl-2 ethanol acrylic and stearyl methacrylate onto polyester fibers using benzoyl peroxide as initiator," Polymer Testing, vol. 21, no. 5, pp. 607-612, 2002.

[12] T. Sun, P. Xu, Q. Liu, J. Xue, and W. Xie, "Graft copolymerization of methacrylic acid onto carboxymethyl chitosan," European Polymer Journal, vol. 39, no. 1, pp. 189-192, 2003.

[13] Z. P. Yao and B. Ranby, "Surface modification by continuous graft copolymerization. III. Photoinitiated graft copolymerization onto poly(ethylene terephthalate) fiber surface," Journal of Applied Polymer Science, vol. 41, no. 7-8, pp. 1459-1467, 1990.

[14] N. Somanathan, B. Balasubramaniam, and V. Subramaniam, "Grafting of Polyester Fibers," Journal of Macromolecular Science-Pure and Applied Chemistry, vol. 32, no. 5, pp. 10251036, 1995.

[15] O. Sanli, S. Aytemiz, and H. L. Unal, "Graft copolymerization of acrylamide on swollen poly(ethylene terephthalate) fibers 
using cerium ammonium nitrate initiator," Journal of Macromolecular Science-Pure and Applied Chemistry, vol. A34, no. 6, pp. 1003-1015, 1997.

[16] N. Inagaki, S. Tasaka, and M. Masumoto, "Improved adhesion between Kapton film and copper metal by plasma graft polymerization of vinylimidazole," Macromolecules, vol. 29, no. 5, pp. 1642-1648, 1996. 

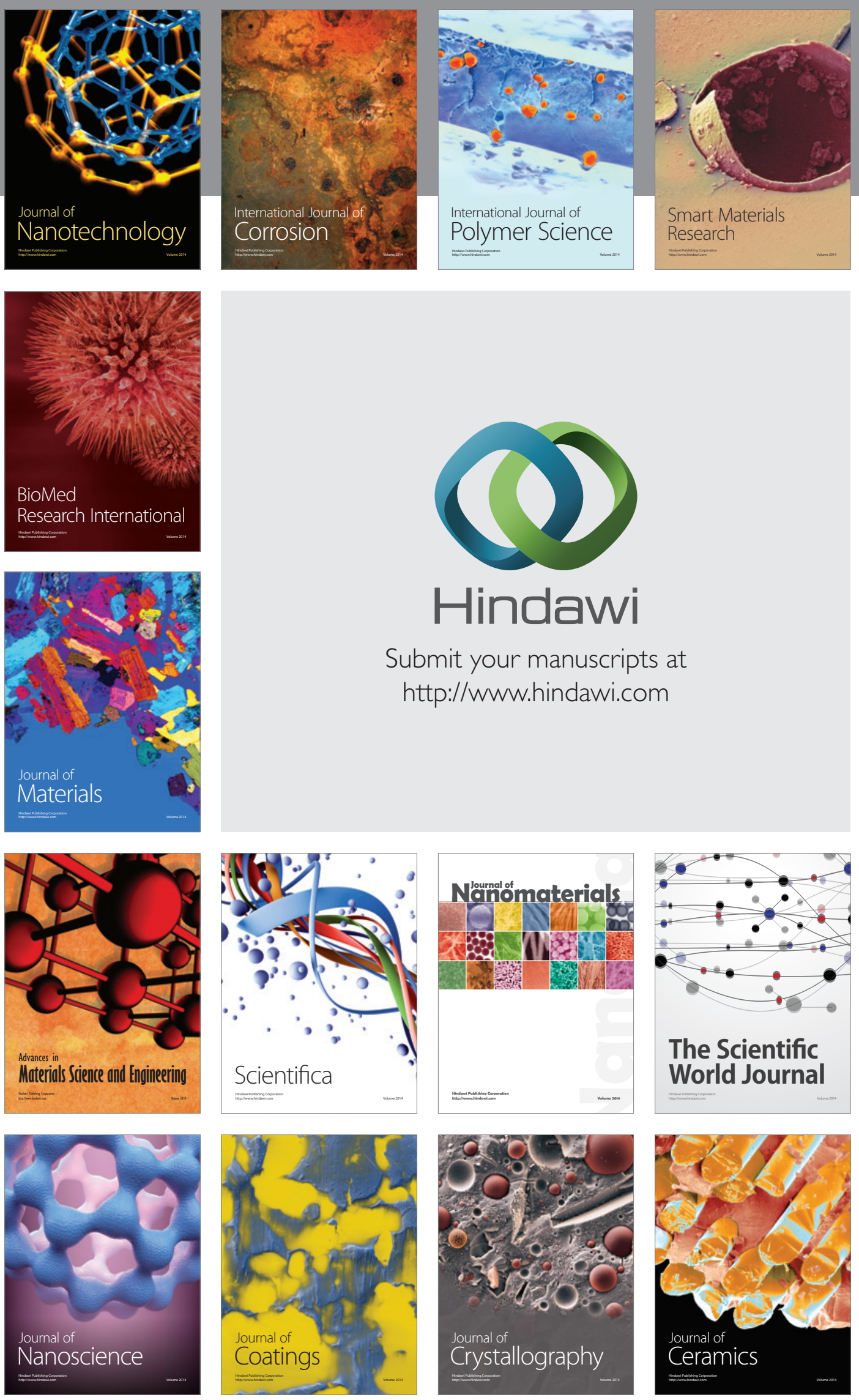

The Scientific World Journal

Submit your manuscripts at

http://www.hindawi.com

\section{World Journal}

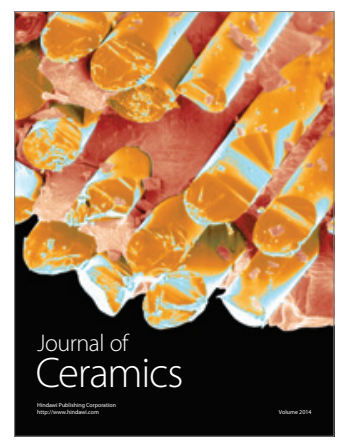

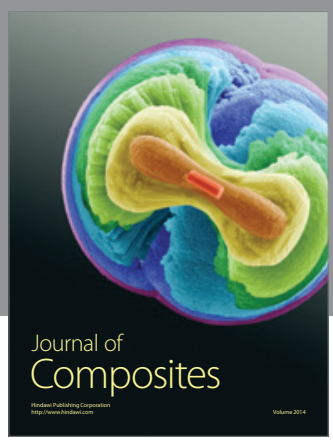
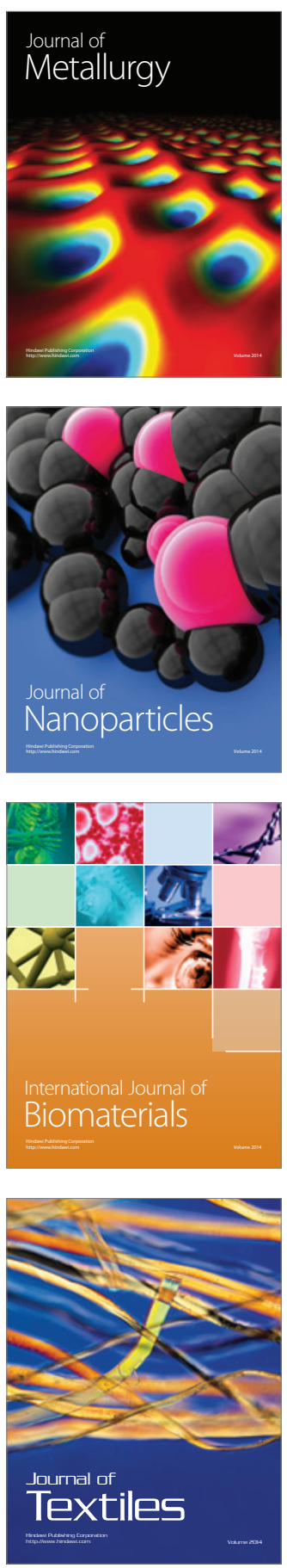